\title{
Aplicación de teorías de la educación a la planificación de una actividad de aprendizaje psicomotor: experiencia con un programa de reanimación neonatal en estudiantes universitarios
}

\author{
Applying educational theories into planning a psychomotor \\ learning activity: an undergraduate neonatal resuscitation \\ programme experience
}

Dr. Erwin J. Khoo, $\mathrm{MRCPCH}^{a}$, Dra. Siew H. Chua, MRCP y Dr. Stefan Kutzsche, M.D. ${ }^{c}$

\section{RESUMEN}

El programa de reanimación neonatal es un buen ejemplodeuna intervención educativa eficazque ha mejorado las tasas de mortalidad perinatal en muchos países. En este artículo, compartimos nuestra experiencia con la planificación de un programa de reanimación neonatal para estudiantes universitarios con fundamento en los principios básicos de las teorías de la educación del currículo en espiral, la taxonomía de Bloom para planificar los resultados del aprendizaje, el modelo de estilos de aprendizaje de Kolb y el marco para la evaluación clínica de Miller. La participación de los médicos clínicos en las teorías pedagógicas podría no estar en línea con la manera en que estos consideraban que aprendían mejor tradicionalmente; aun así, es clave para mejorar el concepto del aprendizaje y los resultados de las intervenciones educativas en el campo de las profesiones relacionadas con la atención médica. Este artículo apunta a ilustrar la aplicación de estas teorías de la educación con un ejemplodela práctica. Estructuramos este artículo en el marco del contenido, la presentación y la evaluación de la planificación de una actividad de aprendizaje psicomotor.

Palabras clave: plan de estudios, aprendizaje, educación médica, facultades de medicina, pedagogía.

http:/ / dx.doi.org/10.5546/aap.2019.e181

Texto completo en inglés:

http:/ / dx.doi.org/10.5546/ aap.2019.eng.e181

Médica

Internacional,

Malasia.

\section{Corrrespondencia:}

Dr. Erwin Khoo: jiayuan_khoo@imu. edu.my

\section{Financiamiento:}

Ninguno.

Conflicto de intereses:

Ninguno que declarar.

Recibido: 22-5-2018

Aceptado: 4-12-2018
El programa de reanimación neonatal (PRN), establecido en 1987 por la Academia Estadounidense de Pediatría y la Asociación Estadounidense del Corazón, se implementó en muchos países como programa de capacitación sistemática para los profesionales de la atención perinatal. En la actualidad, más de dos millones de profesionales de la salud en más de 120 países han recibido capacitación en la técnica de reanimación neonatal para asistir en cada parto. ${ }^{1}$ Gracias a esta intervención educativa, en muchos países se informó una mejora en las tasas de mortalidad perinatal y neonatal hacia la llegada del nuevo milenio. ${ }^{2-4}$

Sin embargo, el PRN apunta principalmente a los profesionales y no así a los estudiantes universitarios. Dada la profundidad de los conocimientos y las habilidades que se necesitan, parece improbable que un médico residente (de primer o segundo año) sea competente con la primera exposición al programa. Asimismo, se sabe que la enseñanza de habilidades psicomotoras es un desafío a nivel universitario debido a los plazos y recursos limitados. ${ }^{5,6}$ Modificamos la estructura del programa original para adaptarlo a los estudiantes del último año de la carrera de medicina de la Universidad Médica Internacional (Malasia) con el objetivo de presentar el PRN ante los estudiantes a modo de proceso (de 
aprendizaje) espiral que les permita prepararse para formar parte de la fuerza laboral. No obstante, el desafío es diseñar e implementar una actividad de aprendizaje psicomotor dentro de los plazos limitados de la carrera de medicina.

En este artículo, se describe nuestra experiencia con la planificación de la actividad según los principios básicos de las teorías de la educación. Se ilustra de qué manera es posible adaptar un programa educativo establecido y diseñado para los profesionales al aprendizaje

Figura 1. Planificación de la introducción del Programa de Reanimación Neonatal. en la Facultad de Medicina en el extremo del currículo en espiral

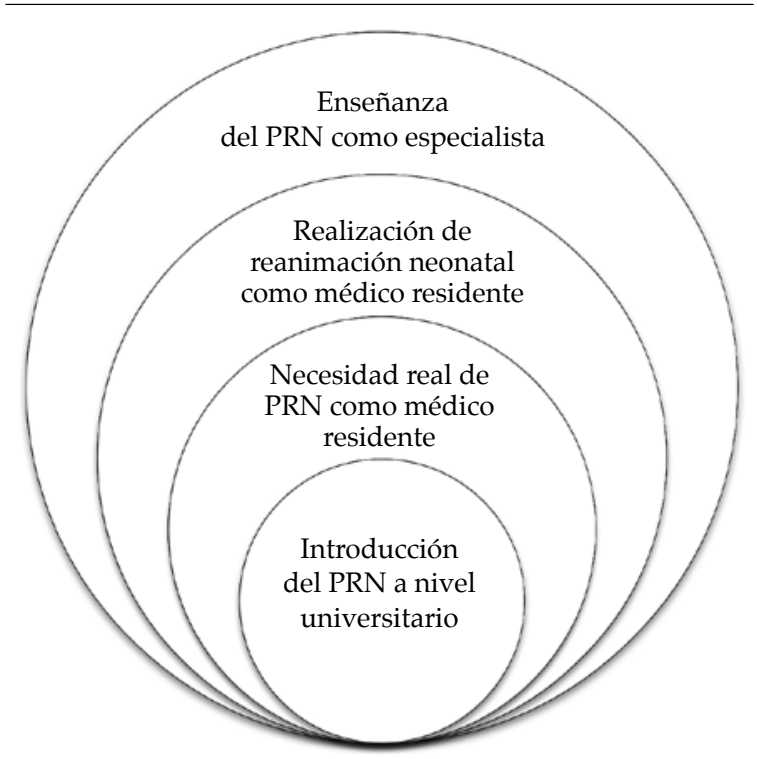

PRN: Programa de reanimación neonatal. de los estudiantes universitarios. Estructuramos este artículo en el marco del plan de estudios, el contenido, la presentación y la evaluación de la planificación de una actividad de aprendizaje psicomotor.

\section{CONTENIDO}

\section{Currículo en espiral}

Los estudiantes universitarios deben reconocer la necesidad del aprendizaje para toda la vida como parte de la educación médica. Los avances tecnológicos y la evolución de Internet dieron como resultado una experiencia de aprendizaje rápida, convirtiendo los años de educación médica en solo el inicio de un currículo en espiral para toda la vida. El currículo en espiral es un concepto descrito por Jerome Bruner ${ }^{7}$ en 1960, que incluye el repaso repetitivo de temas, lo cual requiere profundizar los conocimientos con cada nuevo aprendizaje relacionado con el anterior, junto con un aumento del nivel de dificultad y de la competencia.

Introdujimos el PRN a nuestros estudiantes universitarios durante el último año porque, en ese momento, las experiencias acumuladas durante sus actividades de aprendizaje en la facultad de medicina funcionan como un importante reservorio de experiencias. Este conjunto de habilidades clínicas constituye cimientos importantes sobre los cuales se introduce el aprendizaje psicomotor, que requiere la coordinación de habilidades cognitivas y funciones físicas. En la Figura 1, se describe cómo la presentación del PRN a los estudiantes universitarios encaja en su profesión durante toda la vida.

TABLA 1. Uso de la taxonomía de Bloom para planificar los resultados del aprendizaje

\begin{tabular}{|c|c|c|}
\hline $\begin{array}{l}\text { Taxonomía } \\
\text { de Bloom }\end{array}$ & Definición & $\begin{array}{l}\text { Resultado del aprendizaje por nivel de complejidad: } \\
\text { Hacia el final de este programa, los estudiantes deben ser capaces de... }\end{array}$ \\
\hline Recordar & $\begin{array}{l}\text { Recordar información relevante } \\
\text { ya memorizada. }\end{array}$ & $\begin{array}{l}\text { Recordar el algoritmo estándar para los escenarios de } \\
\text { reanimación neonatal. }\end{array}$ \\
\hline Comprender & $\begin{array}{l}\text { Determinar el significado de los } \\
\text { conceptos pedagógicos. }\end{array}$ & $\begin{array}{l}\text { Interpretar y clasificar los diferentes escenarios de emergencia } \\
\text { que presentan los recién nacidos. }\end{array}$ \\
\hline Aplicar & $\begin{array}{l}\text { Llevar a cabo soluciones prácticas } \\
\text { en una situación específica. }\end{array}$ & $\begin{array}{l}\text { Realizar y demostrar la protección de las vías aéreas, la asistencia } \\
\text { respiratoria, el masaje cardíaco y la administración de fármacos } \\
\text { durante una emergencia. }\end{array}$ \\
\hline Analizar & $\begin{array}{l}\text { Dividir los escenarios en partes y } \\
\text { detectar de qué manera se } \\
\text { relaciona una con la otra. }\end{array}$ & Diferenciar la causa del fracaso de la reanimación. \\
\hline Evaluar & Formular un juicio según el desempeño. & Criticar y valorar las actividades de aprendizaje. \\
\hline Crear & $\begin{array}{l}\text { Combinar elementos para desarrollar } \\
\text { un escenario original. }\end{array}$ & $\begin{array}{l}\text { Reflexionar y producir grupos de escenarios diversos en los } \\
\text { que los estudiantes actúan como profesores. }\end{array}$ \\
\hline
\end{tabular}


La introducción del PRN a los estudiantes universitarios es solo la punta de la espiral. Una vez que se gradúan, los médicos residentes en Malasia reciben el PRN real según les corresponda. La profundización de la competencia continúa cuando los médicos residentes reaniman a un recién nacido durante una emergencia real en su rol de aprendiz competente. Por lo tanto, la exposición previa al PRN es un intento para facilitar la retención de los conocimientos con el refuerzo de dicha información durante el programa estándar más adelante.

\section{Educación basada en los resultados}

Después de comprender la relevancia de introducir una actividad de aprendizaje psicomotor en un programa universitario, la tarea siguiente es que los profesores desarrollen los resultados del aprendizaje previstos de la actividad adecuados a los estudiantes. Este concepto de educación basada en los resultados (outcome based education, OBE) fue fomentado por $\mathrm{Spady}^{8}$ en 1994 como un abordaje basado en el desempeño en el contexto de la educación médica. Los resultados previstos al final del programa deben estar claramente indicados en las etapas de la planificación. Cada vez que un estudiante se gradúa de cada etapa de la espiral, los resultados del aprendizaje en la curva de aprendizaje posterior serían diferentes.

Los resultados del aprendizaje definen el proceso de lo que se piensa. Esto cobra relevancia cuando existen diversos profesores dentro del mismo departamento. Es de ayuda cuando un profesor no está disponible y permite que el profesor sustituto logre los resultados previstos sin modificar el plan de estudios. Si bien los resultados ya están especificados, los medios para lograrlos son bastante amplios. Durante todo el proceso de aprendizaje (y de enseñanza), pueden adaptarse diferentes habilidades de los estudiantes (o de los profesores) y estilos de aprendizaje (o de enseñanza).

El conocimiento de la taxonomía de Bloom es la raíz de la planificación de los resultados del aprendizaje. En 1956, Bloom ${ }^{9}$ clasificó las habilidades de pensamiento por nivel de complejidad con un modelo jerárquico, las que se consideraban procesos de aprendizaje importantes. Luego Anderson y col. ${ }^{10}$ revisaron este modelo en 2001 con verbos que describían la taxonomía (Tabla 1). En la Tabla 2, se describe nuestra experiencia redactando los resultados del aprendizaje según los objetivos SMART (eSpecífico, Medible, Alcanzable, Realista y en Tiempo), que fueron descritos por primera vez por George Doran ${ }^{11}$ en 1981 al redactar metas y objetivos de gestión.

Sin embargo, la OBE ha recibido críticas. ${ }^{12}$ Se la percibe como limitante en una vasta cantidad de campos de la medicina y se considera que el modelo rígido transforma a los profesores en "técnicos de la educación". La OBE también falla al abordar la creatividad, una dimensión importante en el avance de la atención médica. La medicina cambia rápidamente: lo que aprendimos en la facultad de medicina una década atrás podría tornarse obsoleto en la siguiente.

Aun así, la OBE permite que los estudiantes comprendan lo que se espera de ellos y les permite crear su propia agenda de aprendizaje. Los estudiantes pueden reflexionar sobre los contenidos y comprenderlos mejor a su propio ritmo. Se requiere prestar atención y evitar la rigidez al implementar la OBE. La meta máxima es mejorar el aprendizaje de los estudiantes. ${ }^{13}$

TABLA 2. Resultados del aprendizaje redactados según los objetivos SMART

\begin{tabular}{lll}
\hline $\mathbf{S}$ & Específico & Los resultados se detallan explícitamente en la guía para los estudiantes. \\
$\mathbf{M}$ Medible & Se dispone de una evaluación teórica antes y después de la intervención (educativa) y de \\
& & $\begin{array}{l}\text { una lista de verificación del desempeño de las habilidades del PRN para medir los resultados } \\
\text { de los estudiantes. }\end{array}$
\end{tabular}

A Alcanzable Debe ser posible alcanzar los resultados dada la experiencia educativa de los estudiantes a nivel universitario.

R Realista y enfocado en los resultados

Los resultados son relevantes y realistas para los estudiantes conforme al PRN obligatorio para todos los médicos.

$\mathrm{T}$ en Tiempo y ajustado

Los resultados se modifican para que se ajusten a lo que se espera de los estudiantes a nivel universitario.

PRN: Programa de reanimación neonatal. 


\section{Presentación}

Técnica STEPS y modelo de estilos de aprendizaje de Kolb

Un enfoque útil para estructurar una actividad de aprendizaje psicomotor es la técnica STEPS (acrónimo en inglés de establecimiento de la base, demostración del profesor, explicación, práctica bajo supervisión, práctica personalizada posterior $)^{14}$ En 1976, David Kolb ${ }^{15}$ desarrolló un modelo de aprendizaje experiencial para establecer que los elementos del aprendizaje eficaz se observan cuando un estudiante progresa a lo largo de un ciclo de cuatro etapas: (1) tener una experiencia concreta, seguida de (2) una observación reflexiva sobre dicha experiencia, lo que lleva a (3) la formación de conceptos abstractos para el desarrollo de ideas nuevas, que luego (4) se experimentan activamente en situaciones futuras, lo que conlleva a experiencias nuevas. En la Tabla 3, se muestra un ejemplo de cómo emparejamos la técnica STEPS con el modelo de Kolb para enseñar cómo administrar ventilación a presión positiva (VPP).

\section{Educación médica basada en la simulación}

La educación médica basada en la simulación (simulation based medical education, SBME) se define como una actividad educativa que utiliza herramientas o métodos de simulación para crear experiencias nuevas con oportunidades de aprendizaje. La ventaja de la SBME es que permite la realización de comentarios y un aprendizaje activo para los estudiantes, y que fomenta el uso de habilidades para la toma de decisiones en un entorno similar al clínico, a la vez que destaca la seguridad y la protección de

TABLA 3. Uso de la técnica STEPS a fin de enseñar habilidades técnicas para administrar ventilación a presión positiva según el modelo de aprendizaje experiencial de Kolb

\begin{tabular}{|c|c|c|c|c|}
\hline Técnica STEPS & Ciclo de aprendizaje & \multirow{2}{*}{\multicolumn{3}{|c|}{ Estilos de aprendizaje de los estudiantes }} \\
\hline \multicolumn{2}{|c|}{$\begin{array}{l}\text { Establecimiento de la base. El profesor } \\
\text { explica los resultados del aprendizaje y la } \\
\text { relevancia de las habilidades. }\end{array}$} & & & \\
\hline $\begin{array}{l}\text { El profesor } \\
\text { demuestra la } \\
\text { VPP sin otra } \\
\text { explicación. }\end{array}$ & $\begin{array}{l}\text { Experiencia concreta } \\
\text { en la que se enfrenta } \\
\text { una nueva experiencia } \\
\text { con la VPP en los } \\
\text { recién nacidos. }\end{array}$ & \multirow{2}{*}{$\begin{array}{l}\text { Percepción } \\
\text { de la } \\
\text { información: } \\
\text { los } \\
\text { individuos } \\
\text { prefieren } \\
\text { observar y } \\
\text { escuchar en } \\
\text { lugar de } \\
\text { hacer. }\end{array}$} & & \\
\hline $\begin{array}{l}\text { Explicación de la } \\
\text { VPP eficaz junto } \\
\text { con una } \\
\text { demostración. }\end{array}$ & $\begin{array}{l}\text { Observación } \\
\text { reflexiva que permite } \\
\text { reflexionar sobre la } \\
\text { nueva experiencia } \\
\text { enfrentada. }\end{array}$ & & \multirow{2}{*}{$\begin{array}{l}\text { Los } \\
\text { individuos } \\
\text { prefieren } \\
\text { recibir } \\
\text { información } \\
\text { adecuada y } \\
\text { clara y } \\
\text { reflexionar } \\
\text { lógicamente } \\
\text { sobre la } \\
\text { información } \\
\text { brindada. }\end{array}$} & \\
\hline $\begin{array}{l}\text { Los estudiantes } \\
\text { practican la VPP } \\
\text { bajo supervisión } \\
\text { y reciben } \\
\text { comentarios. }\end{array}$ & $\begin{array}{l}\text { La conceptualización } \\
\text { abstracta ocurre } \\
\text { cuando se forman } \\
\text { nuevas ideas con base } \\
\text { en la reflexión. }\end{array}$ & & & $\begin{array}{l}\text { Procesamiento } \\
\text { de la } \\
\text { información: } \\
\text { los individuos } \\
\text { prefieren } \\
\text { resolver } \\
\text { problemas }\end{array}$ \\
\hline $\begin{array}{l}\text { Práctica } \\
\text { personalizada } \\
\text { posterior. }\end{array}$ & $\begin{array}{l}\text { La etapa de } \\
\text { experimentación acti } \\
\text { va tiene lugar cuando } \\
\text { los estudiantes aplican } \\
\text { las ideas nuevas en su } \\
\text { entorno para apreciar } \\
\text { si aparecen cambios la } \\
\text { próxima vez que } \\
\text { atraviesan la } \\
\text { experiencia (Tabla 4). }\end{array}$ & & & $\begin{array}{l}\text { y poner el } \\
\text { aprendizaje en } \\
\text { práctica. }\end{array}$ \\
\hline
\end{tabular}


los intereses de los pacientes. ${ }^{6,16}$ En 2009, Peter Dieckmann ${ }^{17}$ describió los elementos de la SBME con un modelo de ocho fases (Tabla 4). Aplicamos este enfoque en la etapa de "experimentación activa" de los estudiantes, es decir, la parte final del ciclo de aprendizaje de Kolb.

\section{Evaluación}

Al planificar las evaluaciones, es importante estar al tanto del objetivo de la evaluación y dónde encaja en la curva de aprendizaje de los estudiantes. En 1990, la pirámide de George Miller ${ }^{18}$ sirvió para ilustrar el marco de las diferentes facetas de la evaluación

FIGURA 2. Marco para la evaluación clínica de Miller

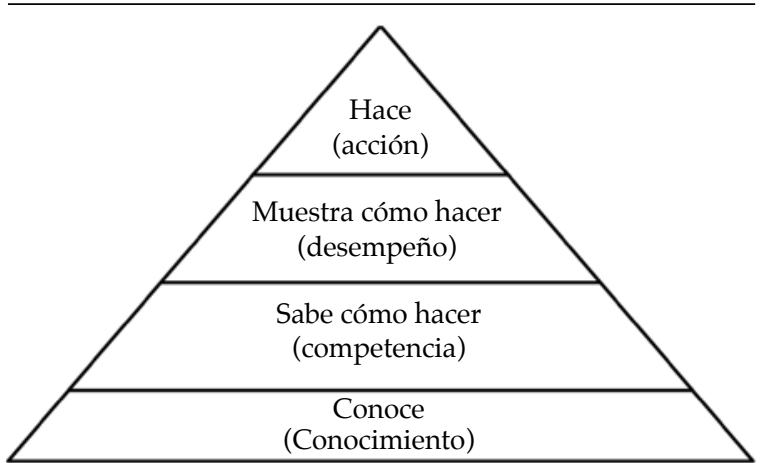

clínica (Figura 2). Nos preguntamos: “¿Estamos evaluando los conocimientos, la competencia, el desempeño o la acción de los estudiantes?". En el nivel inferior y en el extremo de la espiral de aprendizaje, los estudiantes deben conocer qué se necesita para realizar una reanimación neonatal. A nivel universitario, esta base de conocimiento sería el pilar de la evaluación de los estudiantes. Para cumplir el objetivo de su carrera en un futuro, los estudiantes deben saber cómo usar el conocimiento que han acumulado. Esta calidad de ser funcionalmente adecuado define más tarde la competencia como profesional de la atención de la salud aunque no aún como estudiante universitario. La realización de la reanimación neonatal y la enseñanza de las habilidades necesarias para esta se ilustran en la parte superior de la pirámide.

Todos los métodos de evaluación tienen sus propias fortalezas y debilidades..$^{19}$ Las evaluaciones formativas y acumulativas son los dos tipos más frecuentes en la carrera de medicina. Mientras que la evaluación formativa supervisa a los estudiantes y les brinda comentarios durante el proceso de aprendizaje, la evaluación acumulativa examina el aprendizaje de los estudiantes al final del programa en comparación con una referencia. Nuestra evaluación se centró en la "evaluación para el aprendizaje" (formativa) más

TABLa 4. Ocho fases de la educación médica basada en la simulación descritas por Dieckmann y col.

\begin{tabular}{ll}
\hline 1. Introducción & Los profesores son responsables de que la simulación parezca lo más real posible (crear un entorno \\
& realista, usar la mayor cantidad posible de equipos reales y diseñar escenarios que los estudiantes \\
& puedan imaginarse). Debe existir un acuerdo de "dejar de lado la incredulidad" entre el profesor y los \\
& estudiantes, quienes deben hacer su mayor esfuerzo para pretender que todo es real.
\end{tabular}

2. Sesión informativa Familiarización con el simulador, los equipos y el entorno.

con simulador

3. Aporte teórico

4. Sesión informativa sobre el escenario

5. Simulación

y finalización

6. Sesión informativa y comentarios
Presentación de los resultados del aprendizaje.

Por ejemplo, los estudiantes reciben datos sobre el escenario con una parturienta sin complicaciones y un recién nacido que requiere evaluación e intervención.

Los profesores concluyen la presentación del escenario con una declaración imparcial, como por ejemplo, "aquí concluye el escenario; ahora hagamos una sesión informativa." sin brindar su opinión sobre el desempeño de los estudiantes durante la simulación.

Durante la sesión informativa ocurre el verdadero aprendizaje. El PRN universitario comenzó con un comentario positivo que reforzó la buena conducta y el desarrollo de la competencia, a la vez que estableció una atmósfera segura y constructiva. Los estudiantes que cometieron errores tuvieron la oportunidad de contar cómo lo hubieran hecho si hubieran tenido otra chance. El aprendizaje más valioso ocurre cuando los estudiantes reconocen sus errores. A menudo, una sesión informativa eficaz cuestiona a los estudiantes (en lugar de hacerles comentarios). Se pueden usar estrategias como "cuénteme qué sucede cuando no se observa elevación del pecho durante la VPP", "dígame qué causó el desacuerdo" o "¿qué aprendió cuando la saturación no se elevó después de haber intentado la VPP?".

\section{Pausas}

8. Finalización del curso Evaluación del curso y preparación de los planes de aprendizaje. 
que la "evaluación del aprendizaje" (acumulativa).

En vista de la restricción temporal de un plan de estudios de por sí abarrotado, establecimos una evaluación formativa teórica mediante un conjunto de preguntas realizadas con pruebas antes y después del programa. La prueba previa fue un conjunto de preguntas que los estudiantes debían responder antes de la intervención educativa, seguida de una prueba posterior en la que los estudiantes respondieron las mismas preguntas. Si bien las pruebas previas y posteriores se usan tradicionalmente para medir los conocimientos adquiridos después de la intervención, las usamos para involucrar a los estudiantes en el aprendizaje. Este modelo con el aporte de comentarios respalda y mejora el aprendizaje, ya que motiva a los estudiantes a "aprender, no solo estudiar". Con un análisis estadístico que incluyó la prueba $t$ en muestras emparejadas aplicada al muestreo por conveniencia de nuestra cohorte piloto, formada por 28 estudiantes la primera vez que se introdujo la actividad, la media del puntaje en la prueba previa y en la prueba posterior fue de 44,3 (desviación estándar [DE] + 11,2) y 61,6 $(\mathrm{DE}+12,8)$, respectivamente. El puntaje aumentó significativamente por $+17,3 \%$ (DE $+9,2$; intervalo de confianza del $95 \%$ : 13,8-20,9) $(p<0,001)$. Esta metodología puede transferirse a los profesores que desean asumir el desafío de probar la eficacia de una intervención educativa.

\section{LIMITACIONES}

Observamos varias limitaciones con esta experiencia. En primer lugar, se podría argumentar que, para evaluar una actividad de aprendizaje psicomotor, se requiere una herramienta de evaluación "práctica" en lugar de una "escrita". Además, la evaluación "escrita" es un predictor deficiente del desempeño de habilidades de reanimación. ${ }^{20}$ Sin embargo, la evaluación mediante simulación requiere la participación activa del instructor y una proporción de pocos estudiantes por instructor. Esto implica costos ocultos y consume el tiempo de los profesores. Para evitar el agotamiento y mantener la implementación de esta actividad de aprendizaje psicomotor de manera satisfactoria, las actividades incluidas en el plan de estudios universitario deben ser creativas para desarrollar, sostener y gestionar la fuerza laboral a cargo de la capacitación. ${ }^{21}$ Nuestra experiencia demostró que una actividad de aprendizaje psicomotor es factible, rentable y transferible a lo que se espera a nivel universitario. En segundo lugar, los principios educativos aplicados son generales y no específicos del PRN universitario. No queda claro en qué medida los conocimientos adquiridos se tradujeron en una retención real de los conocimientos después de la graduación. Además, tampoco fue posible estimar un tamaño de la muestra significativo porque no existe una definición comúnmente aceptada sobre qué constituye un aprendizaje importante a nivel educativo en términos de habilidades de reanimación neonatal. ${ }^{22}$

\section{CONCLUSIONES}

En este artículo sobre los principios básicos de la educación, se ofrece un marco práctico que los coordinadores de cursos y programas pueden usar en la planificación de las actividades de enseñanza de habilidades psicomotoras destinadas a los estudiantes de la facultad de medicina. La necesidad de actividades de enseñanza de habilidades está bien reconocida, por lo que el desafío es que sean eficaces, sostenibles y rentables para los programas universitarios. A pesar de esto, existe un amplio abismo que debe salvarse entre un modelo de simulación que brinda retroalimentación preprogramada a los estudiantes y un paciente real que se sacudirá y gritará.

En una institución educativa, es fundamental proyectar el plan de estudios universitario de modo tal que los estudiantes se empapen del sentido de un aprendizaje para toda la vida y de una educación adulta madura. Existen varios trabajos que adaptaron el PRN a los estudiantes universitarios, pero el desafío sigue siendo estimar su eficacia. ${ }^{22-25}$ Hemos demostrado que es sencillo aplicar los principios básicos de las teorías de la educación para planificar este tipo de actividad destinada a los estudiantes universitarios. Se requieren estudios futuros con un seguimiento de los graduados que aprovecharon esta experiencia.

\section{REFERENCIAS}

1. American Academy of Pediatrics. Neonatal Resuscitation Program - Home. [Fecha de acceso: 17 de abril de 2018]. Disponible en: https: / www.aap.org/en-us/ continuingmedical-education/life-support/NRP / Pages / History. aspx

2. Boo NY. Neonatal resuscitation programme in Malaysia: an eight-year experience. Singapore Med J. 2009; 50(2): 152-9.

3. Finkelstein JZ, Duhau M, Speranza A. Trend in infant mortality rate in Argentina within the framework of the Millennium Development Goals. Arch Argent Pediatr. 2016; 114(3):216-22. 
4. Patel A, Khatib MN, Kurhe K, Bhargava S, et al. Impact of neonatal resuscitation trainings on neonatal and perinatal mortality: a systematic review and meta-analysis. BMJ Paediatr Open. 2017; 1(1):e000183.

5. Vogel D, Harendza S. Basic practical skills teaching and learning in undergraduate medical education-a review on methodologicalevidence. GMS JMed Educ.2016;33(4):Doc64.

6. Clerihew L, Rowney D, Ker J. Simulation in paediatric training. Arch Dis Child Educ Pract Ed.2016; 101(1):8-14.

7. Bruner JS. The Process of Education. Cambridge, MA: Harvard University Press 1960.

8. Spady WG. Outcome-Based Education: Critical Issues and Answers. Arlington, Va: American Association of School Administrators; 1994.

9. Bloom BS. Taxonomy of educational objectives: The classification of educational goals. New York: Longman; 1956.

10. Anderson LW, Krathwohl DR (eds). A taxonomy for learning, teaching, and assessing: A revision of Bloom's taxonomy of educational objectives. Boston: Allyn \& Bacon; 2001.

11. Doran GT.There'saS.M.A.R.T. way to write management's goals and objectives. Management Rev. 1981; 70(11):35-6.

12. Touchie $\mathrm{C}$, ten Cate $\mathrm{O}$. The promise, perils, problems and progress of competency $\square$ based medical education. Med Educ. 2016; 50(1):93-100.

13. Tam M. Outcomes-based approach to quality assessment and curriculum improvement in higher education. Quality Assurance in Education. 2014; 22(2):158-68.

14. Ker JS. Clinical Skills Centre Teaching. En: Dent J, Harden R (eds). A Practical Guide for Medical Teachers. $4^{\text {th }}$ ed. London: Churchill Livingstone-Elsevier; 2013.Pág.79.

15. Kolb DA. Learning styles inventory. Boston: Hay Group, Hay Resources Direct; 1976.
16. Khoo EJ, Schremmer RD, Diekema DS, Lantos JD. Ethical Concerns When Minors Act as Standardized Patients. Pediatrics.2017; 139(3):e20162795.

17. DieckmannP,DieckmannP.Simulation settingsforlearning in acute medical care. En: Using Simulations for Education, Training and Research. Lengerich:Pabst; 2009.Págs.40-138.

18. Miller GE. The assessment of clinical skills/competence/ performance. Acad Med. 1990; 65(9 Suppl):S63-7.

19. Epstein RM. Assessment in medical education. N Engl J Med. 2007; 356(4):387-96.

20. Rodgers DL, Bhanji F, McKee BR. Written evaluation is not a predictor for skills performance in an Advanced Cardiovascular Life Support Course. Resuscitation. 2010; 81(4):453-6

21. Acton RD, Chipman JG, Lunden M, Schmitz CC. Unanticipated teaching demands rise with simulation training: strategies for managing faculty workload. J Surg Educ. 2015; 72(3):522-9.

22. Lai NM, Ngim CF, Fullerton PD. Teaching medical students neonatal resuscitation: knowledge gained and retained from a brief simulation-based training workshop. Educ Health (Abingdon). 2012; 25(2):105-10.

23. Chimhuya S, Mbuwayesango B, Aagaard EM, Nathoo KJ. Development of a neonatal curriculum for medical students in Zimbabwe-a cross sectional survey. BMC Med Educ. 2018; 18(1):90.

24. Kudreviciene A, Nadisauskiene RJ, Tameliene R, Tamelis A, et al. Initial neonatal resuscitation: skill retention after the implementation of the novel $24 / 7 \mathrm{HybridLab}^{\circledR}$ learning system. J MaternFetal Neonatal Med. 2017; 22:1-8.

25. Nimbalkar A, Patel D, Kungwani A, Phatak A, et al. Randomized control trial of high fidelity vs low fidelity simulation for training undergraduate students in neonatal resuscitation. BMC Res Notes. 2015; 8:636. 